Check for updates

Cite this: RSC Adv., 2019, 9, 1487

27th October 2018

Accepted 2nd January 2019

DOI: $10.1039 / c 8 r a 08909 d$

rsc.li/rsc-advances

\title{
Formal [4 +1] cycloaddition of in situ generated 1,2-diaza-1,3-dienes with diazo esters: facile approaches to dihydropyrazoles containing a quaternary center $\dagger$
}

\author{
Bo Chen, Wen-Dao Chu D * and Quan-Zhong Liu*
}

A Cu(II)/bisoxazoline ligand-promoted formal [4 + 1] cycloaddition of diazo esters with azoalkenes formed in situ has been developed. This strategy provides a potential protocol for the construction of dihydropyrazoles containing a quaternary center with good to excellent yields.

The efficient construction of quaternary carbon centers has remained a crucial issue in organic synthesis. ${ }^{1}$ Quaternary carbon centers are ubiquitous in various natural products, and pharmaceutically relevant compounds. ${ }^{2}$ Although significant efforts have been devoted to the effective construction of quaternary centers in recent years, ${ }^{1}$ new methodologies that could be advantageous in terms of functional-group tolerance, operational simplicity, and the use of easily obtained starting materials are still highly desired.

On the other hand, dihydropyrazoles represent a class of important heterocycles that occur in biologically active natural products and pharmaceuticals such as anti-amoebic, hypotensive, analgesic, anti-bacterial, anti-cancer, anti-depressant and nonsteroidal anti-inflammatory agents. ${ }^{3}$ Accordingly, great research efforts have been devoted toward their synthesis, and remarkable advances have been achieved in the construction of these nitrogen heterocycles. Representative synthetic strategies include formal $[3+2]$ cycloaddition, ${ }^{4}[4+$ 1] cycloaddition, ${ }^{5}$ catalytic asymmetric Fischer's pyrazoline synthesis via a sequential aza-Michael addition/ cyclocondensation process, ${ }^{6}$ and photocatalytic radical cyclization. ${ }^{7,8}$ In comparison with the more ubiquitous family of $[3$ $+2]$ cycloadditions, $[4+1]$ cycloannulations are relatively underutilized in these target-directed five-membered azaheterocycles construction. ${ }^{5}$ In 2012, Bolm and coworkers reported the first example of asymmetric synthesis of dihydropyrazoles by formal $[4+1]$ cycloaddition of in situ derived azoalkenes and sulfur ylides (Scheme 1a). ${ }^{5 a}$ Recently, diazo

Chemical Synthesis and Pollution Control, Key Laboratory of Sichuan Province, College of Chemistry and Chemical Engineering, China West Normal University, No. 1, Shida Road, Nanchong 637002, P. R. China. E-mail: chuwendaonpo@126.com; quanzhongliu@cwnu.edu.cn

$\dagger$ Electronic supplementary information (ESI) available: Experimental procedures and compound characterisation data, including X-ray crystal structures of $\mathbf{3 h}$. CCDC 1840892. For ESI and crystallographic data in CIF or other electronic format see DOI: $10.1039 / \mathrm{c} 8 \mathrm{ra} 08909 \mathrm{~d}$ esters as 1,1-dipolar C1 synthons had also been utilized by the group of Favi to synthesize racemic dihydropyrazoles in a similar manner (Scheme 1b). ${ }^{5 b}$ However, none of these investigations has explored the possibility of accessing dihydropyrazoles containing a quaternary center. Herein, we present a $\mathrm{Cu}(\mathrm{II}) /$ bisoxazoline ligand-promoted formal $[4+1]$ cycloaddition of diazo esters with azoalkenes formed in situ, affording dihydropyrazoles containing a quaternary center with good to excellent yields (Scheme 1c).

At the outset of this investigation, we employed hydrazone 1a and diazo ester 2a as the substrates (Table 1). Preliminary screening showed that the ligand has a remarkable effect on the reaction. For instance, the reaction with phosphine ligands gave the desired dihydropyrazole $3 \mathbf{a}$ in low yields (Table 1, entry 2-4). It was found that the reaction proceeded efficiently when bisoxazoline L6 was employed as ligand, leading to the desired product $3 \mathbf{a}$ in $98 \%$ yield (Table 1, entry 7). Subsequently, different bases and solvents were then explored (Table 1, entries 7-16), $\mathrm{Na}_{2} \mathrm{CO}_{3}$ and $\mathrm{CH}_{2} \mathrm{Cl}_{2}$ was the best choice.

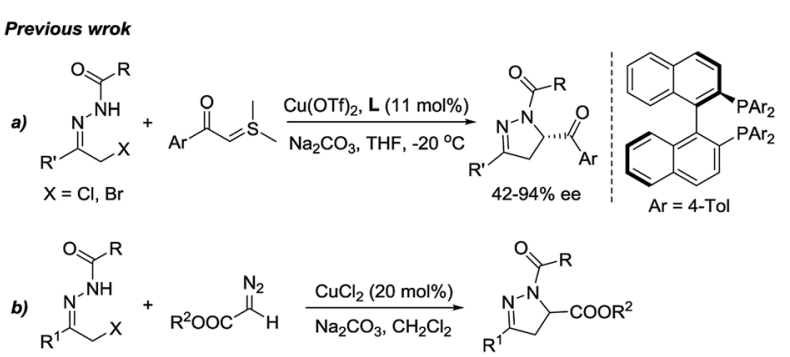

This wrok

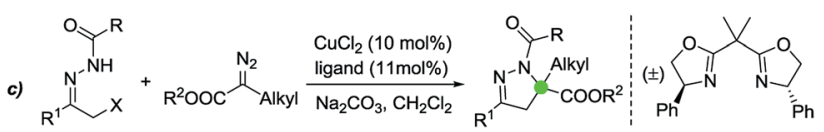

Scheme 1 Synthesis of dihydropyrazoles by formal $[4+1]$ cycloaddition. 
Table 1 Optimization of reaction conditions ${ }^{a}$

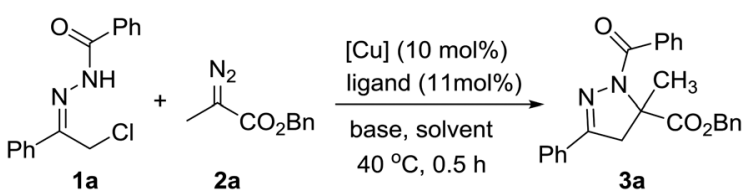

\begin{tabular}{llllll}
\hline Entry & {$[\mathrm{Cu}]$} & Ligand & Base & Solvent & Yield $\left.{ }^{b} \%\right)$ \\
\hline 1 & $\mathrm{CuCl}_{2}$ & None & $\mathrm{Na}_{2} \mathrm{CO}_{3}$ & $\mathrm{CH}_{2} \mathrm{Cl}_{2}$ & None \\
2 & $\mathrm{CuCl}_{2}$ & $\mathbf{L 1}$ & $\mathrm{Na}_{2} \mathrm{CO}_{3}$ & $\mathrm{CH}_{2} \mathrm{Cl}_{2}$ & 18 \\
3 & $\mathrm{CuCl}_{2}$ & $\mathbf{L 2}$ & $\mathrm{Na}_{2} \mathrm{CO}_{3}$ & $\mathrm{CH}_{2} \mathrm{Cl}_{2}$ & 6 \\
4 & $\mathrm{CuCl}_{2}$ & $\mathbf{L 3}$ & $\mathrm{Na}_{2} \mathrm{CO}_{3}$ & $\mathrm{CH}_{2} \mathrm{Cl}_{2}$ & 22 \\
5 & $\mathrm{CuCl}_{2}$ & $\mathbf{L 4}$ & $\mathrm{Na}_{2} \mathrm{CO}_{3}$ & $\mathrm{CH}_{2} \mathrm{Cl}_{2}$ & 5 \\
6 & $\mathrm{CuCl}_{2}$ & $\mathbf{L 5}$ & $\mathrm{Na}_{2} \mathrm{CO}_{3}$ & $\mathrm{CH}_{2} \mathrm{Cl}_{2}$ & 6 \\
7 & $\mathrm{CuCl}_{2}$ & $\mathbf{L 6}$ & $\mathrm{Na}_{2} \mathrm{CO}_{3}$ & $\mathrm{CH}_{2} \mathrm{Cl}_{2}$ & 98 \\
8 & $\mathrm{CuCl}_{2}$ & $\mathbf{L 6}$ & $\mathrm{K}_{2} \mathrm{CO}_{3}$ & $\mathrm{CH}_{2} \mathrm{Cl}_{2}$ & 15 \\
9 & $\mathrm{CuCl}_{2}$ & $\mathbf{L 6}$ & $\mathrm{Cs}_{2} \mathrm{CO}_{3}$ & $\mathrm{CH}_{2} \mathrm{Cl}_{2}$ & 26 \\
10 & $\mathrm{CuCl}_{2}$ & $\mathbf{L 6}$ & $\mathrm{NaOH}_{2}$ & $\mathrm{CH}_{2} \mathrm{Cl}_{2}$ & Trace \\
11 & $\mathrm{CuCl}_{2}$ & $\mathbf{L 6}$ & $\mathrm{KO}^{2} \mathrm{Bu}_{2}$ & $\mathrm{CH}_{2} \mathrm{Cl}_{2}$ & Trace \\
12 & $\mathrm{CuCl}_{2}$ & $\mathbf{L 6}$ & $\mathrm{Et}_{3} \mathrm{~N}_{2}$ & $\mathrm{CH}_{2} \mathrm{Cl}_{2}$ & Trace \\
13 & $\mathrm{CuCl}_{2}$ & $\mathbf{L 6}$ & $\mathrm{Na}_{2} \mathrm{CO}_{3}$ & $\mathrm{THF}_{2}$ & 83 \\
14 & $\mathrm{CuCl}_{2}$ & $\mathbf{L 6}$ & $\mathrm{Na}_{2} \mathrm{CO}_{3}$ & $\mathrm{Toluene}_{2}$ & Trace \\
15 & $\mathrm{CuCl}_{2}$ & $\mathbf{L 6}$ & $\mathrm{Na}_{2} \mathrm{CO}_{3}$ & $\mathrm{CH}_{3} \mathrm{CN}_{2}$ & 5 \\
16 & $\mathrm{CuCl}_{2}$ & $\mathbf{L 6}$ & $\mathrm{Na}_{2} \mathrm{CO}_{3}$ & $\mathrm{Hexane}^{2}$ & 12
\end{tabular}

${ }^{a}$ Reaction was run under the following conditions: a solution of $1 \mathrm{a}(0.1$ $\mathrm{mmol}), 2 \mathrm{a}(0.5 \mathrm{mmol})$, base $(0.5 \mathrm{mmol}), \mathrm{Cu}$ cat. $(10 \mathrm{~mol} \%)$, and ligand $(11 \mathrm{~mol} \%)$ in anhydrous solvent $(1 \mathrm{~mL})$ was stirred at $40{ }^{\circ} \mathrm{C}$ under nitrogen atmosphere for $0.5 \mathrm{~h} .{ }^{b}$ Yields refer to isolated products.

With the optimized conditions in hand, we next explored the substrate scope of the heterodienes. A series of hydrazones 1a-1 bearing electron-neutral, -deficient or -rich aromatic substituents were smoothly reacted with diazo ester $\mathbf{2 a}$ to give the corresponding dihydropyrazoles 3a-1 in 76-98\% yield (Table 2, entry 112). Also $\alpha$-bromo $N$-benzoyl hydrazone 10 reacted well, and $88 \%$ yield were achieved (Table 2, entry 15). In contrast, 2-naphthylsubstituted hydrazone $\mathbf{1 m}$ and aliphatic hydrazone $1 \mathrm{n}$ only gave a small quantity of product $3 \mathbf{m}$ and $3 \mathbf{n}$ (Table 2, entry 13-14).

Next, the scope of the reaction was extended by conducting the reaction with various diazo esters (Table 3). Variation of the ester $\mathrm{R}^{2}$ group (entries 1 and 2) had little influence on the yield of product 3. The significant steric effect of $\mathrm{R}^{1}$ has been observed. Methyl and ethyl groups gave excellent results (entries $2-3$ ), while the more bulky groups gave only a trace of products (entries 4-5).

We next attempted to investigate asymmetric variant of this $\mathrm{Cu}(\mathrm{II})$-catalyzed formal $[4+1]$ cycloaddition reaction of diazo esters with azoalkenes formed in situ (Scheme 2). An extensive screening of chiral phosphine ligands (L7, L8), bisoxazoline ligands (L9-12) and different reaction conditions had been implemented. Unfortunately, only up to $5 \%$ ee was obtained when L12 was employed as chiral ligand, albeit with excellent yield (98\%).

To show the synthetic potential of this strategy, we have carried out a gram scale synthesis of 3 a (Scheme 3). Under the optimized reaction conditions, the reaction with $3 \mathrm{mmol}$ of $\mathbf{1 a}$
Table 2 Substrate scope for hydrazones ${ }^{a}$

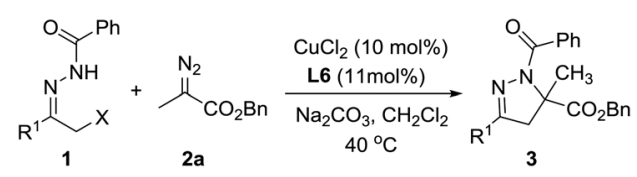

\begin{tabular}{|c|c|c|c|c|}
\hline Entry & 1 & $\mathrm{x}$ & $\mathrm{R}^{1}$ & Yield $^{b}$ of $3(\%)$ \\
\hline 1 & $1 a$ & $\mathrm{Cl}$ & $\mathrm{Ph}$ & $3 a, 98$ \\
\hline 2 & $1 b$ & $\mathrm{Cl}$ & $2-\mathrm{Br}-\mathrm{Ph}$ & $3 b, 82$ \\
\hline 3 & $1 \mathrm{c}$ & $\mathrm{Cl}$ & $2-\mathrm{F}-\mathrm{Ph}$ & $3 \mathbf{c}, 78$ \\
\hline 4 & $1 d$ & $\mathrm{Cl}$ & $2-\mathrm{CH}_{3}-\mathrm{Ph}$ & $3 d, 76$ \\
\hline 5 & $1 e$ & $\mathrm{Cl}$ & 3-Cl-Ph & $3 e, 93$ \\
\hline 6 & 1f & $\mathrm{Cl}$ & $3-\mathrm{OCH}_{3}-\mathrm{Ph}$ & 3f, 92 \\
\hline 7 & $1 \mathrm{~g}$ & $\mathrm{Cl}$ & $3-\mathrm{CH}_{3}-\mathrm{Ph}$ & $3 g, 89$ \\
\hline 8 & $1 \mathrm{~h}$ & $\mathrm{Cl}$ & 4-Cl-Ph & $3 \mathbf{h}, 98$ \\
\hline 9 & $1 \mathrm{i}$ & $\mathrm{Cl}$ & $4-\mathrm{F}-\mathrm{Ph}$ & $3 \mathbf{i}, 94$ \\
\hline 10 & $1 \mathrm{j}$ & $\mathrm{Cl}$ & $4-\mathrm{OCH}_{3}-\mathrm{Ph}$ & $3 \mathbf{j}, 98$ \\
\hline 11 & $1 \mathrm{k}$ & $\mathrm{Cl}$ & $4-\mathrm{NO}_{2}-\mathrm{Ph}$ & $3 \mathbf{k}, 92$ \\
\hline 12 & 11 & $\mathrm{Cl}$ & $4-\mathrm{CH}_{3}-\mathrm{Ph}$ & 31,98 \\
\hline 13 & $1 \mathrm{~m}$ & $\mathrm{Cl}$ & 2-Naphthyl & $3 \mathbf{m}$, trace \\
\hline 14 & $1 n$ & $\mathrm{Cl}$ & $n$-Bu & $3 n$, trace \\
\hline 15 & 10 & $\mathrm{Br}$ & $\mathrm{Ph}$ & 30,88 \\
\hline
\end{tabular}

${ }^{a}$ Reaction was run under the following conditions: a solution of 1 (0.1 $\mathrm{mmol}), 2 \mathrm{a}(0.5 \mathrm{mmol}), \mathrm{Na}_{2} \mathrm{CO}_{3}(0.5 \mathrm{mmol}), \mathrm{CuCl}_{2}(10 \mathrm{~mol} \%)$, and $\mathbf{L 6}$ (11 mol\%) in anhydrous $\mathrm{CH}_{2} \mathrm{Cl}_{2}(1 \mathrm{~mL})$ was stirred at $40{ }^{\circ} \mathrm{C}$ under nitrogen atmosphere for $0.5 \mathrm{~h} .{ }^{b}$ Yields refer to isolated products.

Table 3 Substrate scope for diazo esters ${ }^{a}$

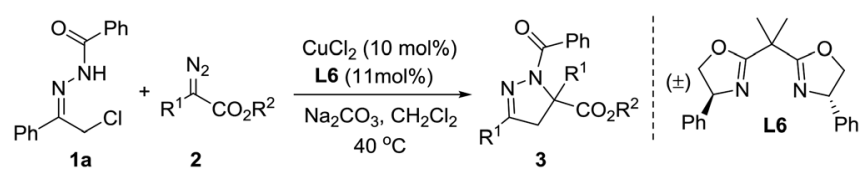

\begin{tabular}{lllll}
\hline Entry & $\mathbf{2}$ & $\mathrm{R}^{1}$ & $\mathrm{R}^{2}$ & Yield $^{b}$ of 3 (\%) \\
\hline 1 & $\mathbf{2 a}$ & $\mathrm{Me}$ & $\mathrm{Bn}$ & $\mathbf{3 a}, 98$ \\
2 & $\mathbf{2 b}$ & $\mathrm{Me}$ & $\mathrm{Et}$ & $\mathbf{3 p}, 98$ \\
3 & $\mathbf{2 c}$ & $\mathrm{Et}$ & $\mathrm{Et}$ & $\mathbf{3 q}, 92$ \\
4 & $\mathbf{2 d}$ & $\mathrm{Bn}$ & $\mathrm{Bn}$ & $\mathbf{3 r}$, trace \\
5 & $\mathbf{2 e}$ & $\mathrm{Ph}$ & $\mathrm{Et}$ & $\mathbf{3 s}$, trace
\end{tabular}

${ }^{a}$ Reaction was run under the following conditions: a solution of $1 \mathrm{a}(0.1$ $\mathrm{mmol}), 2$ (0.5 mmol), $\mathrm{Na}_{2} \mathrm{CO}_{3}(0.5 \mathrm{mmol}), \mathrm{CuCl}_{2}(10 \mathrm{~mol} \%)$, and $\mathbf{L 6}$ (11 mol\%) in anhydrous $\mathrm{CH}_{2} \mathrm{Cl}_{2}(1 \mathrm{~mL})$ was stirred at $40{ }^{\circ} \mathrm{C}$ under nitrogen atmosphere for $0.5 \mathrm{~h} .{ }^{b}$ Yields refer to isolated products.

proceeded smoothly with 5 equiv. of $2 \mathrm{a}$, affording $1.07 \mathrm{~g}$ of $3 \mathrm{a}$ ( $90 \%$ yield).

In summary, we have developed a $\mathrm{Cu}(\mathrm{II}) / \mathrm{bisoxazoline} \mathrm{ligand-}$ promoted formal $[4+1]$ cycloaddition of diazo esters with azoalkenes formed in situ, affording dihydropyrazoles containing a quaternary center with good to excellent yields. The reaction involves the use of stable, readily available starting materials and is operationally simple. 


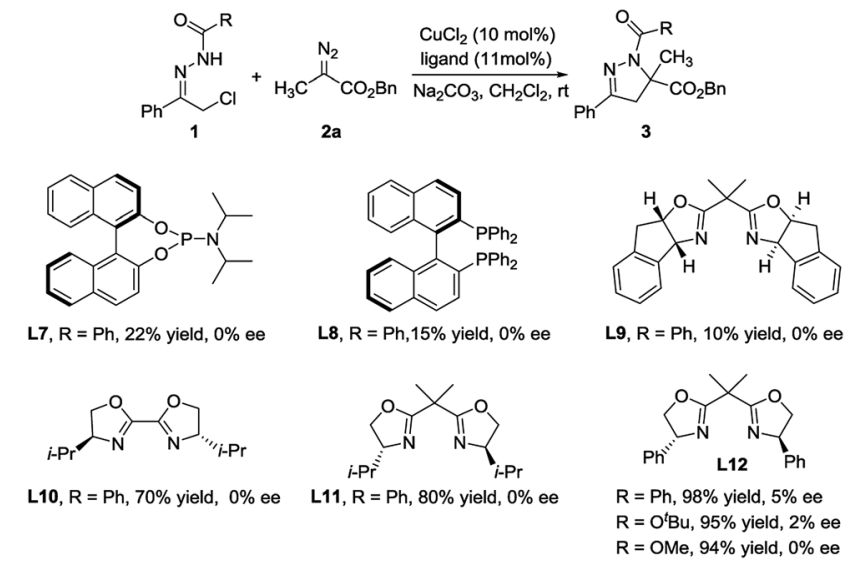

Scheme 2 The investigation on asymmetric $[4+1]$ annulation reaction.

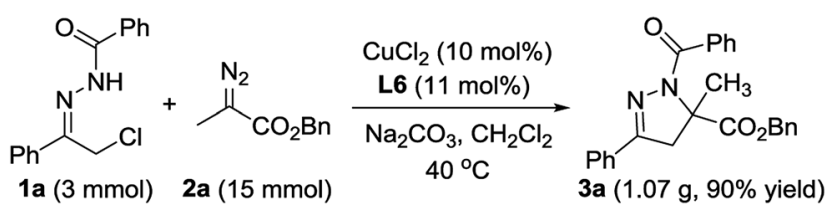

Scheme 3 Reaction on the gram scale.

\section{Conflicts of interest}

There are no conflicts to declare.

\section{Acknowledgements}

This work was financial supported by the National Natural Science Foundation of China (No. 21572183 and 21801208).

\section{Notes and references}

1 For selected reviews, see: (a) K. Fuji, Chem. Rev., 1993, 93, 2037-2066; (b) J. C. Douglas and L. E. Overman, Proc. Natl. Acad. Sci. U. S. A., 2004, 101, 5363-5367; (c) J. Christoffers and A. Baro, Quaternary Stereocenters-Challenges and Solutions for Organic Synthesis, Wiley-VCH, Weinheim, 2005; (d) B. M. Trost and C. Jiang, Synthesis, 2006, 369-396; (e) M. Shimizu, Angew. Chem., Int. Ed., 2011, 50, 5998-6000; $(f)$ B. M. Wang and Y. Q. Tu, Acc. Chem. Res., 2011, 44, 12071222; (g) J. P. Das and I. Marek, Chem. Commun., 2011, 47, 4593-4623; (h) M. Büschleb, S. Dorich, S. Hanessian, D. Tao, K. B. Schenthal and L. E. Overman, Angew. Chem., Int. Ed., 2016, 55, 4156-4186.

2 (a) D. J. Newman and G. M. Cragg, J. Nat. Prod., 2012, 75, 311335; (b) D. J. Newman and G. M. Cragg, J. Nat. Prod., 2016, 79, 629-661 and references within; (c) Y. Tu, C. Jeffries, H. Ruan, C. Nelson, D. Smithson, A. A. Shelat, K. M. Brown, X.-C. Li, J. P. Hester, T. Smillie, I. A. Khan, L. Walker, K. Guy and B. Yan, J. Nat. Prod., 2010, 73, 751-754.
3 (a) M. Kissane and A. R. Maguire, Chem. Soc. Rev., 2010, 39, 845-883; (b) C.-H. Küchenthal and W. Maison, Synthesis, 2010, 719-740; (c) A. Sahoo, S. Yabanoglu, B. N. Sinha, G. Ucar, A. Basu and V. Jayaprakash, Bioorg. Med. Chem. Lett., 2010, 20, 132-136; (d) M. Johnson, B. Younglove, L. Lee, R. LeBlanc, H. Holt Jr, P. Hills, H. Mackay, T. Brown, S. L. Mooberry and M. Lee, Bioorg. Med. Chem. Lett., 2007, 17, 5897-5901; (e) M. A. Ali and M. Shaharyar, Bioorg. Med. Chem., 2007, 15, 1896-1902; $(f)$ J. H. M. Lange and C. G. Kruse, Curr. Opin. Drug Discovery Dev., 2004, 7, 498.

4 (a) S. Kanemasa and T. Kanai, J. Am. Chem. Soc., 2000, 122, 10710-10711; (b) R. Shintani and G. C. Fu, J. Am. Chem. Soc., 2003, 125, 10778-10779; (c) M. P. Sibi, L. M. Stanley and C. P. Jasperse, J. Am. Chem. Soc., 2005, 127, 8276-8277; (d) A. Suárez, C. W. Downey and G. C. Fu, J. Am. Chem. Soc., 2005, 127, 11244-11245; (e) T. Kano, T. Hashimoto and K. Maruoka, J. Am. Chem. Soc., 2006, 128, 2174-2175; (f) M. P. Sibi, L. M. Stanley and T. Soeta, Adv. Synth. Catal., 2006, 348, 2371-2375; (g) M. P. Sibi, L. M. Stanley and T. Soeta, Org. Lett., 2007, 9, 1553-1556; (h) L. Gao, G. S. Hwang, M. Y. Lee and D. H. Ryu, Chem. Commun., 2009, 5460-5462; ( $i$ ) H. Suga, Y. Furihata, A. Sakamoto, K. Itoh, Y. Okumura, T. Tsuchida, A. Kakehi and T. Baba, J. Org. Chem., 2011, 76, 7377-7387; (j) T. Arai and Y. Ogino, Molecules, 2012, 17, 6170-6178; (k) T. Imaizumi, Y. Yamashita and S. Kobayashi, J. Am. Chem. Soc., 2012, 134, 20049-20052; (l) M. Rueping, M. S. Maji, H. B. Kücük and I. Atodiresei, Angew. Chem., Int. Ed., 2012, 51, 1286412868; (m) G. Wang, X. Liu, T. Huang, Y. Kuang, L. Lin and X. Feng, Org. Lett., 2013, 15, 76-79; (n) A. L. Gerten, M. C. Slade, K. M. Pugh and L. M. Stanley, Org. Biomol. Chem., 2013, 11, 7834-7837; (o) T. Arai, Y. Ogino and T. Sato, Chem. Commun., 2013, 49, 7776-7778; (p) T. Hashimoto, Y. Takiguchi and K. Maruoka, J. Am. Chem. Soc., 2013, 135, 11473-11476; (q) M. Hori, A. Sakakura and K. Ishihara, J. Am. Chem. Soc., 2014, 136, 13198-13201; (r) X. Hong, H. B. Kücük, M. S. Maji, Y.-F. Yang, M. Rueping and K. N. Houk, J. Am. Chem. Soc., 2014, 136, 13769-13780; (s) X. Wang, Y.-m. Pan, X.-c. Huang, Z.-y. Mao and H.-s. Wang, Org. Biomol. Chem., 2014, 12, 2028-2032; $(t)$ D.-Y. Zhang, L. Shao, J. Xu and X.-P. Hu, ACS Catal., 2015, 5, 5026-5030.

5 (a) J.-R. Chen, W.-R. Dong, M. Candy, F.-F. Pan, M. Jörres and C. Bolm, J. Am. Chem. Soc., 2012, 134, 6924-6927; (b) O. A. Attanasi, L. D. Crescentini, G. Favi, F. Mantellini, S. Mantenuto and S. Nicolini, J. Org. Chem., 2014, 79, 83318338; (c) Z. Wang, Y. Yang, F. Gao, Z. Wang, Q. Luo and L. Fang, Org. Lett., 2018, 20, 934-937.

6 (a) H. Yanagita and S. Kanemasa, Heterocycles, 2007, 71, 699709; (b) S. Müller and B. List, Angew. Chem., Int. Ed., 2009, 48, 9975-9978; (c) S. Müller and B. List, Synthesis, 2010, 2010, 2171-2178; (d) O. Mahé, I. Dez, V. Levacher and J.-F. Brière, Angew. Chem., Int. Ed., 2010, 49, 7072-7075; (e) N. R. Campbell, B. Sun, R. P. Singh and L. Deng, Adv. Synth. Catal., 2011, 353, 3123-3128; ( $f$ ) M. Fernández, E. Reyes, J. L. Vicario, D. Badía and L. Carrillo, Adv. Synth. Catal., 
2012, 354, 371-376; (g) O. Mahé, I. Dez, V. Levacher and J.-F. Brière, Org. Biomol. Chem., 2012, 10, 3946-3954.

7 (a) X.-Q. Hu, J.-R. Chen, Q. Wei, F.-L. Liu, Q.-H. Deng, A. M. Beauchemin and W.-J. Xiao, Angew. Chem., Int. Ed., 2014, 53, 12163-12167; (b) Q. Wei, J.-R. Chen, X.-Q. Hu, X.-C. Yang, B. Lu and W.-J. Xiao, Org. Lett., 2015, 17, 44644467; (c) J. Cheng, P. Xu, W. Li, Y. Cheng and C. Zhu, Chem. Commun., 2016, 52, 11901-11904; (d) Q.-Q. Zhao, J. Chen, D.-M. Yan, J.-R. Chen and W.-J. Xiao, Org. Lett., 2017, 19,
3620-3623; (e) J.-m. Yu, G.-P. Lu and C. Cai, Chem. Commun., 2017, 53, 5342-5345.

8 For other methods for synthesis of dihydropyrazoles, see: $(a)$ C. B. Tripathi and S. Mukherjee, Org. Lett., 2014, 16, 33683371; (b) X. Wu, M. Wang, G. Zhang, Y. Zhao, J. Wang and H. Ge, Chem. Sci., 2015, 6, 5882-5890; (c) M.-N. Yang, D.-M. Yan, Q.-Q. Zhao, J.-R. Chen and W.-J. Xiao, Org. Lett., 2017, 19, 5208-5211; (d) J. Zhao, M. Jiang and J.-T. Liu, Org. Chem. Front., 2018, 5, 1155-1159. 